\title{
A STEP-Compliant Intelligent Process Planning System for Milling
}

\author{
Ouyang Hua-bing ${ }^{*}$
}

School of Mechanical Engineering, Shanghai Dianji University, Shanghai, 201306, China

\begin{abstract}
The new standard ISO 14649 provides CAD/CAPP/CAM/CNC with an interface and a comprehensive high level and detailed manufacturing information. CNC technology based on STEP-NC will be the future trend, and the intelligent process planning is the core of STEP-NC oriented CNC technology. The objective of this research is to present the architecture, key technologies and implementation of STEP-NC oriented intelligent computer-aided process planning system. Some key technologies, such as feature recognition, setup planning, operations selection, tool selection, machine tool selection and process planning optimization are described in detail. A hybrid approach based on artificial intelligent techniques (neural networks, fuzzy logic and genetic algorithm) is adopted as the inference engine in the proposed system. Based on Solidworks CAD platform, the corresponding prototype system was developed using VB.NET programming language. The implementation of the proposed ST-ICAPP system is demonstrated by an example case study. The result shows that the proposed system is valid and feasible.
\end{abstract}

Keywords: STEP-NC, Feature recognition, Artificial intelligence, CAPP, Neural network.

\section{INTRODUCTION}

Process planning specifies what raw materials are needed to cut, and what processes and operations are necessary to transform those raw materials into the final product. It is the bridge between part design and manufacturing. The outcome of process planning is the information of manufacturing processes and their corresponding parameters, the identification of machine tools, and the fixtures required to perform those processes.

However, it is commonly recognized that ISO 6983 standard has become a bottleneck for the advancement of $\mathrm{CNC}$ manufacturing because of the data non-compliance through $\mathrm{CAD} / \mathrm{CAPP} / \mathrm{CAM} / \mathrm{CNC}$ chain [1]. To eliminate this problem, a new standard known as STEP-NC has been developed since the late 1990s, which is formalized as an ISO 14649 [2]. As a replacement interface of a new data between $\mathrm{CAD} / \mathrm{CAPP} / \mathrm{CAM} / \mathrm{CNC}$ (CAx for short), the main objectives of STEP-NC are aimed at providing CAx with an interface and a comprehensive manufacturing data model. In addition, STEP-NC also provides an opportunity and challenge to promote the improvement of manufacturing capability utilizing high level and detailed information.

Recently, many researches involving STEP-NC interoperability have been carried out. Research in terms of manufacturing technology and processes began with a proposal for the conceptual framework for designing and implementing an intelligent $\mathrm{CNC}$ system by Suh and Sheon [3]. Newman proposed a STEP-compliant CAD/CAM prototype system based on a framework using new ISO 14649 standards for milling components $[4,5]$. To optimize machining

*Address correspondence to this author at the No. 88 Wenjing Road, Shanghai, 200245, China; Tel: 15000600258; E-mail: oyhuabing@163.com parameters, Firman proposed a system framework for machining optimization based on STEP-NC, which consists of an optimization module, a process control module and a knowledge based evaluation module [6]. A STEP-compliant process planning and manufacturing and its implementation have been presented by Xu and Suh [7]. A STEP-compliant framework that makes use of self-learning algorithms has also been studied by Kumar and the pocket and hole features for milling has been tested and certified in the proposed system [8]. Liu et al. proposed a NC programming system for prismatic parts, and the system consisted of three functional modules, namely a feature-based modeler, a process planner and a part program generator. The system can read the STEP-NC file and then calculate the tool path automatically [9]. Many researchers presented the prototype systems to support data interoperability between various CAx systems based on ISO standard 14649 [10-12].

The paper aims at proposing a new intelligent CAPP system based on STEP-NC (called ST-ICAPP for short). STICAPP integrates the design and process planning aspects of manufacturing, utilizing STEP-NC standards and artificial intelligent techniques. ST-ICAPP tries to get a physical STEP-NC file containing the part manufacturing information, search the most suitable setup order and acquire the optimal process route considering many related manufacturing parameters. The proposed methodology involves the development of an intelligent computer-aided process planning procedure to study the machining features recognition, setup planning, and process planning optimization.

\section{FRAMEWORK OF ST-ICAPP SYSTEM}

This section discusses the proposed ST-ICAPP system, which is based on STEP-NC data model and the intelligent 


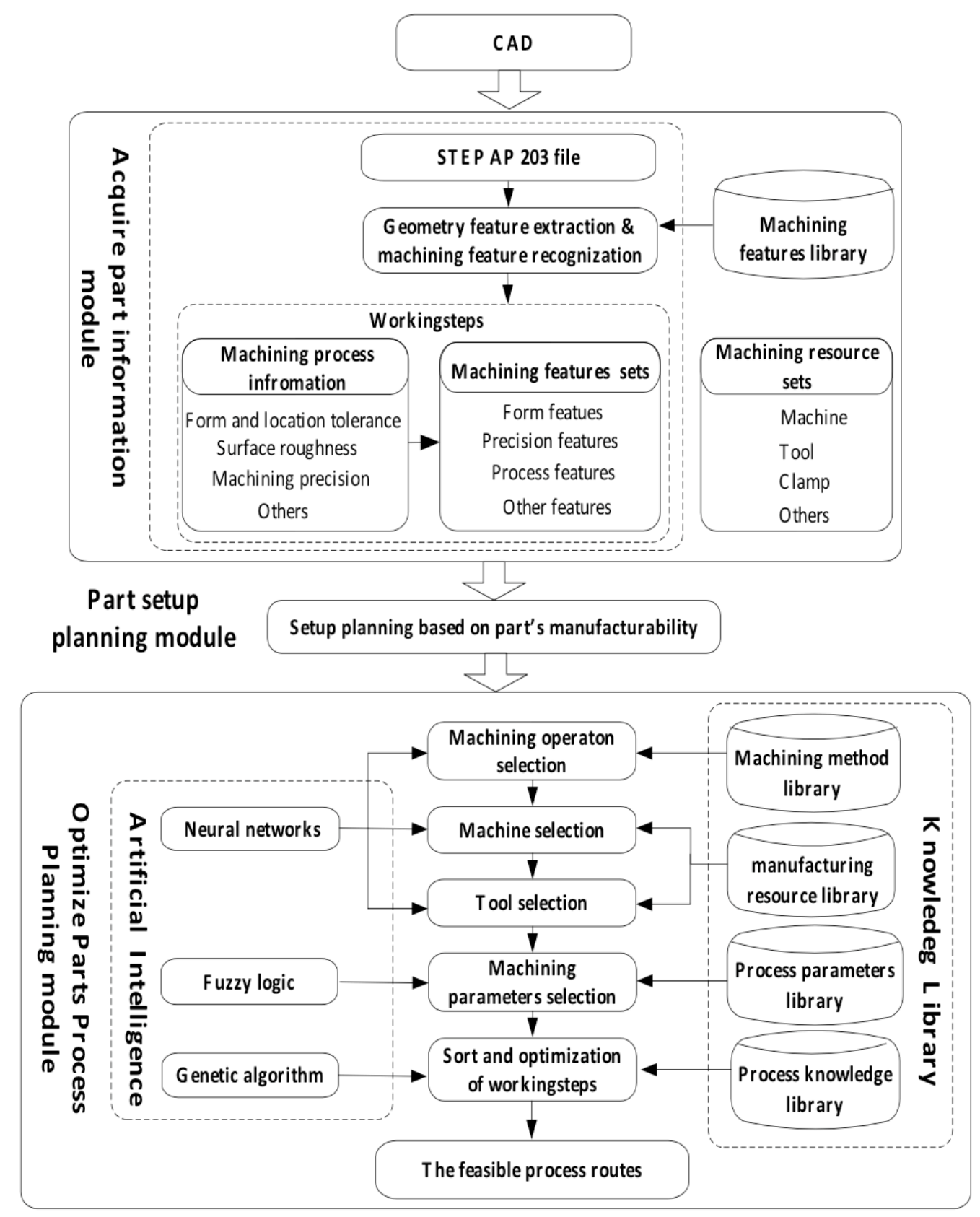

Fig. (1). Framework of the proposed ST-ICAPP system.

computation strategy. The framework of ST-ICAPP is given in Fig. (1). ST-ICAPP can transform a product model into a process plan. These activities are divided into four major hierarchy modules, namely Acquire Part Information, Part Setup Planning, Optimize Parts Process Planning and STEPNC Machining Program.

Acquire Part Information module is responsible for generating part information including the features information and their processes. Part Setup Planning module can group all recognized features with the same or similar setup for machining them into a setup. Optimize Parts Process Planning module is used to generate process planning based on information from Acquire Part Information module and interfaces with process parameter libraries.
In the proposed framework, a part model can be firstly created by Solidworks or other CAD. Manufacturing features of a part design model are recognized by Part Information Acquisition module and then those features are mapped into STEP-NC feature data and its associated attributes. A detailed description on how ST-ICAPP components will be given in the following sections.

\section{KEY TECHNOLOGIES OF ST-ICAPP}

The process planning is the action of preparing detailed machining operations to transform an engineering design to a final functional work piece. The detailed planning contains the route, actions, machining parameters, machines and tools 


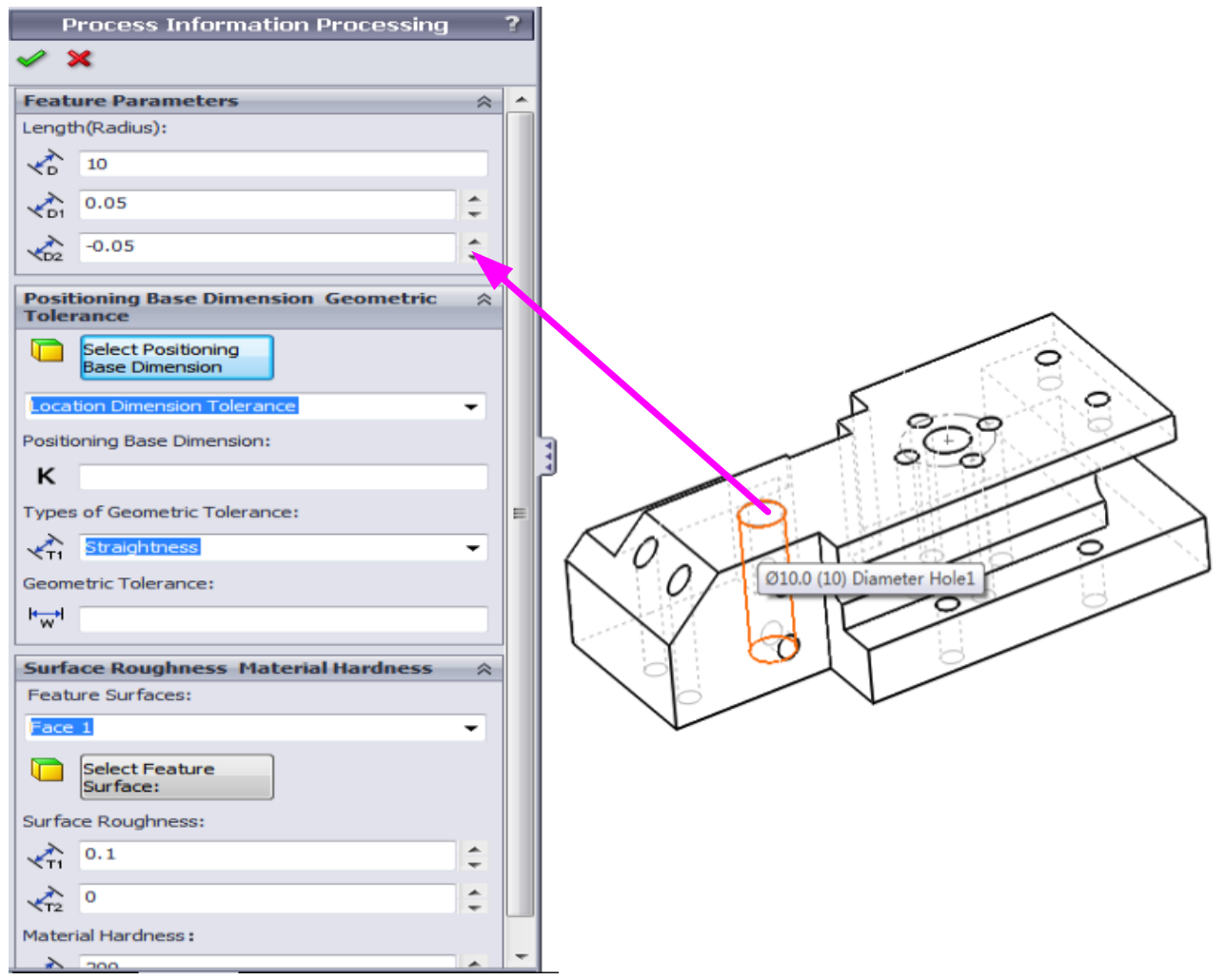

Fig. (2). Process information processing of a selected hole.

for machining a part. In ST-ICAPP system, design entities are transformed into manufacturing features in accordance with STEP-NC standard, and then a process planning is inverted into a process of machining operations related to all generated manufacturing features. Some key technologies of ST-ICAPP will be described in the following.

\subsection{Part Information Acquisition}

Acquire Part Information module consists of two submodules, namely, feature recognition and process information processing. Through Feature recognizer module, the given design part is extracted and then the manufacturing features in accordance with ISO 14649 standards are generated. Feature recognition is considered to be a prerequisite for process planning activities. Feature recognition algorithm determines the performance of ST-ICAPP system. In the recent years, various feature recognition methodologies have been proposed [13-16]. The objective of this research is to develop a STEP-NC oriented form feature extraction system, which converts design data into relevant manufacturing data. The effort is to keep feature recognition as general as possible so that the data can be formed into any STEP schema. The extracted features can be saved in the STEP AP 224 format. In the present work, the STEP-based feature recognition algorithm draws from the algorithm proposed by Mangesh [17].

However, those generated machining features cannot be used directly in the downstream CAPP or CAM applications. It is needed to attach process information to those features by
Process Information Processing module. The process information includes mainly three categories namely: textual attributes, tolerances attributes and process attributes. Tolerances attributes can be attached by clicking the recognized feature. The tolerance values indicate the permissible deviations of a dimension, which can be later used in the process planning. Surface attributes include two important attributes, namely surface finish and surface property. The module provides a process property and can be defined by process name, process parameter, and the unit of measure used. The process can be done through a user interface designed for each feature. Fig. (2) shows the process information processing of a selected hole feature.

\subsection{Part Setup Planning Based on Manufacturability}

A setup planning can be defined as a group of features machined during a single clamping. It is obvious that the goal of setup planning is maximum number of features in minimum number of setups [14]. In fact, clamping and unclamping of the part in the same machine tool affects the accuracy of final part significantly.

The setup planning in ST-ICAPP is composed of three steps: setup generation, operation sequence, and sequencing the setups [18]. The setup generation is a procedure to group the machining operations into setups, so the manufacturing features which have common approach directions are grouped into the same setup. The operation sequence arranges the machining operations in each generated setup into an order. In addition, the cutting tool changes among the 


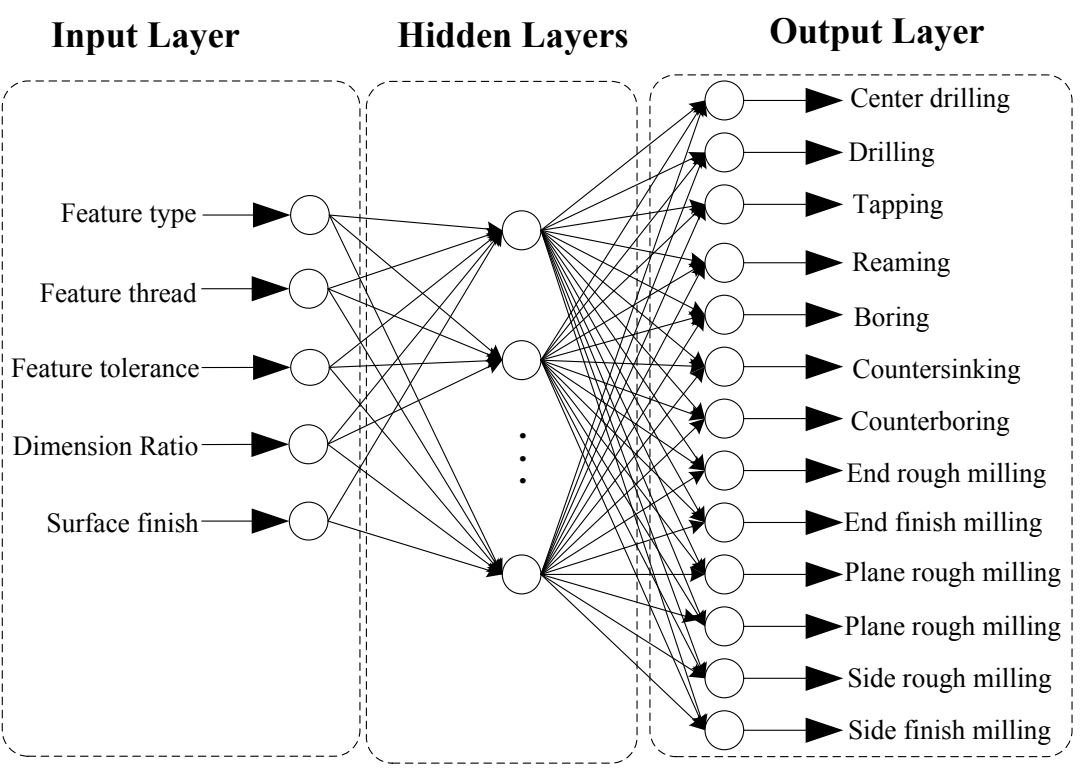

Fig. (3). Neural network model for machining operations selection.

operations are reduced to a minimum. Sequencing the setups arranges the generated setups into an order, so setups with fewer number of machining features are machined firstly. A set of rules deciding on the setup planning are used for prismatic parts [18].

The following steps illustrate the implemented algorithm to generate the required setup planning [19]:

Step 1: Define a part coordinate system and assign tool access direction (TAD) for a part machined on a 3-axis milling.

Step 2: Define six setup plans corresponding to each TAD: $\quad S_{+x}$ for $T A D_{1}, S_{-x}$ for $T A D_{2}, S_{+y}$ for $T A D_{3}, S_{-y}$ for $T A D_{4}$, $S_{+z}$ for $T A D_{5}, S_{-z}$ for $T A D_{6}$.

Step 3: Assign a definite TAD to every feature.

Step 4: Sequence the machining operations on the basis of machining features precedence [19].

Step 5: Arrange machining operations according to the natural operation sequence.

Step 6: Rearrange the machining operations in the light of the following sequence: center drilling $->$ drilling$>$ counterboring or countersinking $->$ tapping $->$ boring or reaming or milling.

Step 7: Minimize the number of tool changes by rearranging same type machining operations.

Step 8: Sequence the setup plans according to the rules that setups with less machining features are machined firstly.

\subsection{Optimization of the Process Planning}

After generating setup planning sequences, validity of their sequences must be further checked by clustering constraints [19]. The optimized sequence for STEP-NC machining operation of a given part, the optimized selection of the machine, cutting tool and machining parameters can then be obtained by the proposed intelligent algorithms containing artificial neural networks, fuzzy logic, and Genetic Algorithm (GA). The proposed intelligent algorithm used in STICAPP system will be subsequently described.

\subsubsection{Application of Artificial Neural Networks}

The proposed ST-ICAPP system uses three neural networks to carry out some tasks of the process planning. These tasks are summarized as follows: selection of machining operations, selection of cutting tool and selection of machine tools.

(1) Selection of machining operations

This task receives data for each feature of a given part and generates the needed machining operations to machine the features. Fig. (3) shows the neural network used in selecting the machining operations. The network consists of three connected layers, namely, the input layer, the output layer and the hidden layers. The input layer consists of five input variables. The hidden layers have 15 neurons, which are decided by means of a number of experiments. The output layer contains thirteen variables corresponding to the machining operations required for machining each generated feature. Each output neuron has a value of 0 or 1 . If the output neuron value is equal to 1 , it means that the selection of the machining operation is supported.

The input values to the network corresponding to the feature type and feature thread are properly encoded to be interpreted by the neural network. The other input variables corresponding to feature dimension ratio, feature tolerance, and feature surface finish are scaled appropriately to be in the range between 0 and 1 in order to facilitate the training of the network.

\section{(2) Selection of cutting tools}

The neural network model for cutting tools selection is mainly used to select the proper cutting tool for each ma- 


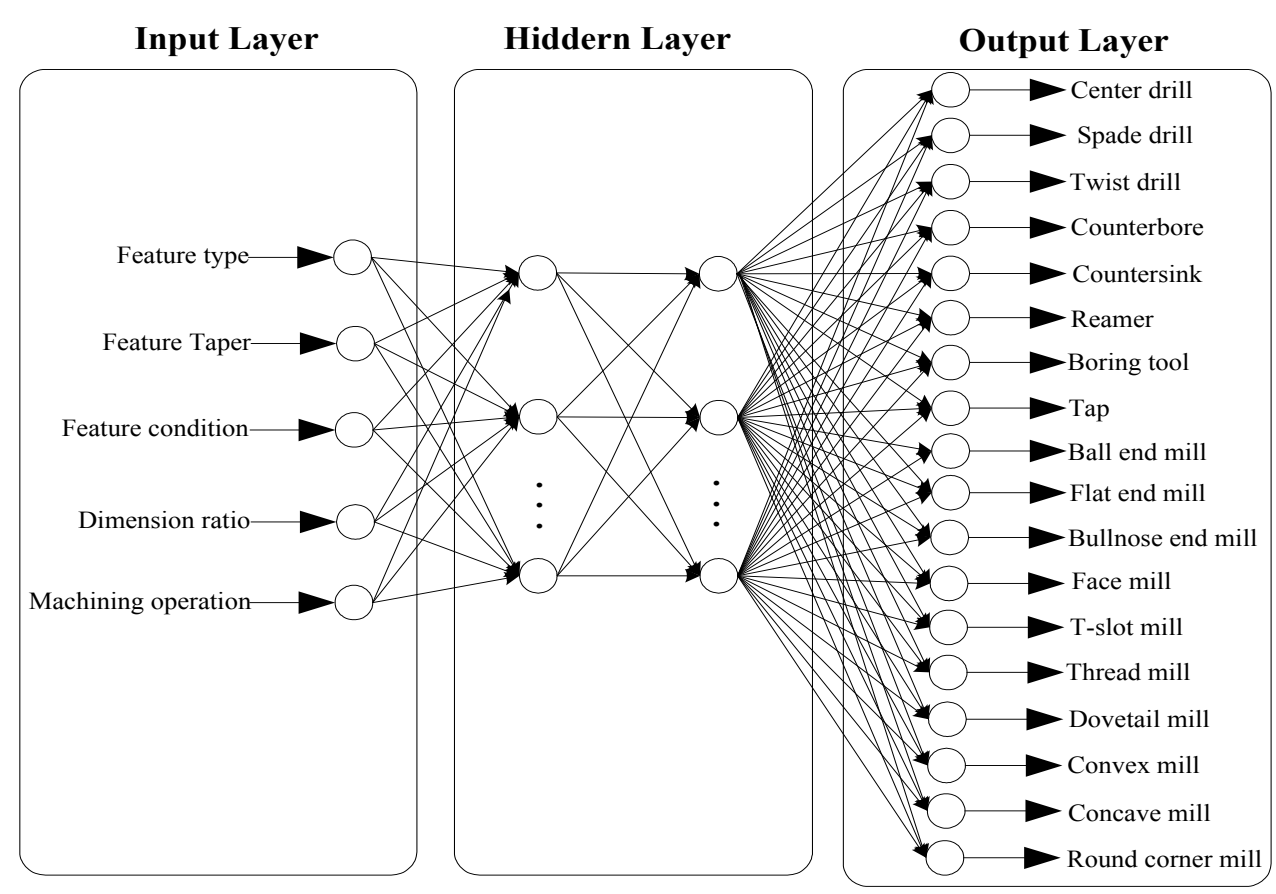

Fig. (4). Neural network model for cutting tools selection.

chining feature [20]. The selection is based on machining feature and its associated machining operation or STEP-NC Workingsteps. The basic strategy in selection of cutting tools is that each Workingstep has a corresponding cutting tool to be used to generate that feature. For instance, Square slot corresponding to an end milling operation, a flat end mill can be selected, while round slot corresponding to the same machining operation, a ball end mill should be adopted. Based on the above criterion, the neural network is trained. The neural network model for cutting tool selection is presented in Fig. (4). The network consists of five input variables, two hidden layers with fifteen neurons and eighteen output variables. The input variables include feature type, feature taper, feature condition, dimension ratio and machining operation. The input values are appropriately encoded and scaled to facilitate the network training. The output variables are the tools corresponding to the cutting tool types. Each output variable has a value of 0 or 1 . If the output variable value is equal to 1 , it means that the selection of the cutting tool is supported. The cutting tools library used in the proposed CAPP system is based on STEP-NC (ISO14649) standard [2]. When the cutting tool is selected by the neural network, the CAPP system starts searching the standard tool dimensions database to find the proper tool dimensions to fit the machining operation.

\section{(3) Selection of machine tools}

Similar to the network of cutting tool selection, the network of machine tools selection is presented in Fig. (5). The input layer includes machining part characteristics (i.e. part type, part dimension and surface finishes) and machining operation characteristics (i.e. machining operation and machining power). The output layer contains recommended parameters of the selected machine tool to perform the task.

\subsubsection{Application of Fuzzy Logic}

A fuzzy logic model is proposed to select machining parameters in drilling and milling operations. There are three basic components of the fuzzy model, namely fuzzification of the input, fuzzy rules application and defuzzification of the output [21]. A set of fuzzy rules has been developed for different work and tool materials combinations. The choice of defuzzification method may have a significant impact on the accuracy of the fuzzy model output. The most frequently used one is the centroid or center of area (COA) which is used in the developed fuzzy models.

For example, the fuzzy model for drilling is designed and the fuzzy sets of the input and output variables are shown in Table 1.

The input variables contain material hardness, thole diameter, cutting depth and pitch. The output variables are cutting speed and feed rate. The universe of input, material hardness, hole diameter, cutting depth and pitch has been partitioned according to the minimum and maximum values allowed to control the model. In the same way, the universe of output, cutting speed and feed rate has been partitioned according to the required range for each output.

\subsubsection{Application of Genetic Algorithm}

To ensure that the generated process planning is optimal or near-optimal, the proposed GA is then presented to implement the process planning optimization. The proposed GA is described as follows [22]:

\section{Step 1: Coding strategy}

A gene in a string represents an operation ID and a corresponding machine tool, cutting tool and tool access direction 
Table 1. Fuzzy sets of input and output variables.

\begin{tabular}{|c|c|c|c|c|c|c|c|c|c|}
\hline \multirow{2}{*}{ Fuzzy Sets } & \multicolumn{3}{|c|}{ Range } & \multirow{2}{*}{ Abbrev. } & \multirow{2}{*}{ Fuzzy Sets } & \multicolumn{3}{|c|}{ Range } & \multirow{2}{*}{ Abbrev } \\
\hline & $\mathbf{a}$ & B & c & & & $\mathbf{a}$ & b & c & \\
\hline \multicolumn{10}{|c|}{ Input variables } \\
\hline \multicolumn{5}{|c|}{ Material Hardness(BHN) } & \multicolumn{5}{|c|}{ Hole diameter(mm) } \\
\hline Very soft & 0 & 0 & 150 & VS & Very small & 0 & 0 & 6 & VS \\
\hline Soft & 0 & 150 & 250 & S & Small & 0 & 6 & 13 & $\mathrm{~S}$ \\
\hline Medium & 150 & 250 & 350 & M & Medium & 6 & 13 & 26.5 & M \\
\hline Hard & 250 & 350 & 450 & $\mathrm{H}$ & Large & 13 & 26.5 & 55 & $\mathrm{~L}$ \\
\hline Very hard & 350 & 450 & 550 & VH & Very large & 26.5 & 55 & 80 & VL \\
\hline \multicolumn{5}{|c|}{ Cutting Depth(mm) } & \multicolumn{5}{|c|}{$\operatorname{Pitch}(\mathrm{mm})$} \\
\hline Very small & 0 & 0 & 1 & VS & Very short & 0 & 0 & 0.5 & VS \\
\hline Small & 0 & 1 & 2.5 & S & Short & 0 & 0.5 & 1.5 & $\mathrm{~S}$ \\
\hline Medium & 1 & 2.5 & 4 & M & Medium & 0.5 & 1.5 & 2.5 & M \\
\hline Large & 2.5 & 4 & 6 & $\mathrm{H}$ & Long & 1.5 & 2.5 & 3.5 & $\mathrm{~L}$ \\
\hline Very large & 4 & 6 & 10 & VL & Very long & 2.5 & 3.5 & 4.5 & VL \\
\hline \multicolumn{10}{|c|}{ Output variables } \\
\hline \multicolumn{5}{|c|}{ Cutting speed(m/min) } & \multicolumn{5}{|c|}{ Feed $\operatorname{rate}(\mathrm{mm} / \mathrm{rev})$} \\
\hline Very low & 0 & 0 & 15 & VL & Very slow & 0 & 0 & 0.15 & VS \\
\hline Low & 0 & 15 & 55 & $\mathrm{~L}$ & Slow & 0 & 0.15 & 0.3 & $\mathrm{~S}$ \\
\hline Medium & 15 & 55 & 100 & M & Medium & 0.15 & 0.3 & 0.8 & M \\
\hline High & 55 & 100 & 160 & $\mathrm{H}$ & Fast & 0.3 & 0.8 & 1.2 & $\mathrm{~F}$ \\
\hline Very high & 100 & 160 & 250 & VH & Very fast & 0.8 & 1.2 & 1.5 & $\mathrm{VF}$ \\
\hline
\end{tabular}

Table 2. Representation of a process plan.

\begin{tabular}{|c|c|c|c|c|c|}
\hline Op3 & Op2 & Op4 & Op6 & Op1 & M01 \\
\hline \hline M04 & M01 & M03 & M02 & T01 & T03 \\
\hline T02 & T03 & T04 & T02 & +y & $-\mathrm{y}$ \\
\hline$-\mathrm{x}$ & $+\mathrm{y}$ & $-\mathrm{z}$ & $-\mathrm{y}$ & & \\
\hline
\end{tabular}

(TAD). Sequence of operations is represented by the order of the genes in the string. Table 2 shows the representation of a six-operation process plan. Op3 represents operation 3; M04, T02 and $-\mathrm{x}$ in the other rows represent the machine, tool and TAD, respectively, which are used to perform operation 4 , so are the other columns.

\section{Step 2: Population initialization}

To get chromosomes, the feasible operation sequences are adopted. Once a number of the feasible operation sequences are assigned, the procedures of population initialization are given as: 


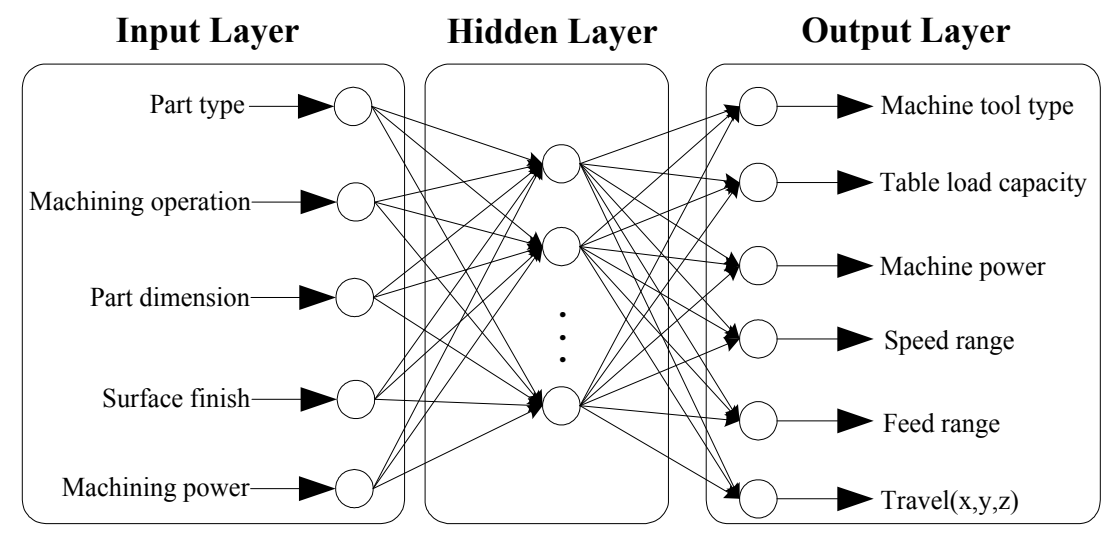

Fig. (5). Neural network model for machine tools selection.

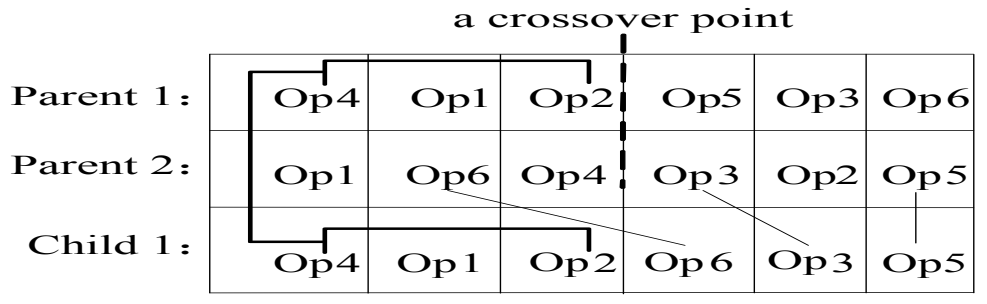

Fig. (6). Crossover change example.

(a) Randomly select one sequence from the available feasible sequences of the operations list.

(b) Visit the first selected operation.

(c) Randomly select machines and tools that can be used for manufacturing the operation.

(d) Randomly select one amongst all possible TADs for the operation.

(e) Repeat steps (c) and (d), until each operation has been assigned a machine, a tool and a TAD.

(f) Repeat steps (a)-(e) until the feasible sequences of the operations are finished.

\section{Step 3: Fitness function}

The fitness function is used to express the adaptability of a string which is expressed by the fitness value. The optimization constraints are often considered as the additive constraint aggregation. These constraints mean that some target functions must be met in the technologic sequence decision, such as minimum processing times, minimum production cost and so on. In this work, the minimum production cost is employed to calculate the fitness of each operation sequence and to measure the efficiency of a manufacturing system. The total production cost is made up of Machine Cost (MC), Tool Cost (TC), Machine Change Cost (MCC), Tool Change Cost (TCC) and Setup Change Cost (SCC). The fitness function is calculated for each individual in the population as described in Eq. (1).

Fitness $=M C+T C+T C C+S C C$
Step 4: Produce a new generation population

(1) Reproduction

The most of reproduction methods, namely roulette wheel's extensions, scaling techniques, tournaments, elitist models, and ranking methods were adopted for numerical optimization [19]. The main objective is to reduce the sampling error and improve calculation precision. When using GA for operations sequencing, the natural number format is used for coding. Compared with other selection operators, the "tournament selection" is more suitable for the problem of operations sequencing and it is adopted in this work.

\section{(2) Crossover}

There are three crossover operators for path representation: Partially Mapped Crossover (PMX), Order Crossover (OX) and Cycle Crossover (CX) [23]. The aim of PMX crossover is keeping the important similarities of parent and child generation. The OX crossover emphasizes that the sequence order is very important. The $\mathrm{CX}$ reserves the absolute position of the elements in the parent generation. Generally, OX is $11 \%$ better than PMX and $15 \%$ better than CX. In this work, an OX crossover operator is adopted to ensure the local precedence of operations is met and a feasible offspring is generated. In order to clarify the crossover operation, a crossover change example is given in Fig. (6).

The procedure of crossover operations is described as follows:

(a) Based on the chromosome length, a crossover point is randomly generated. Each string is then divided into two 


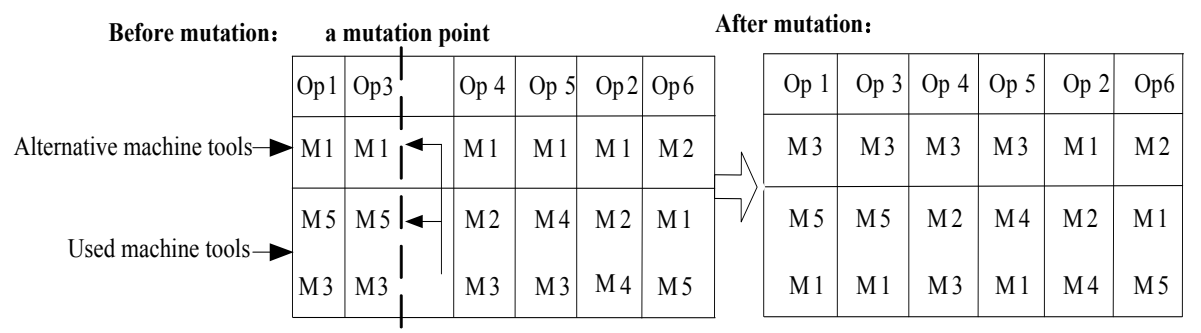

Fig. (7). Mutation operator example.

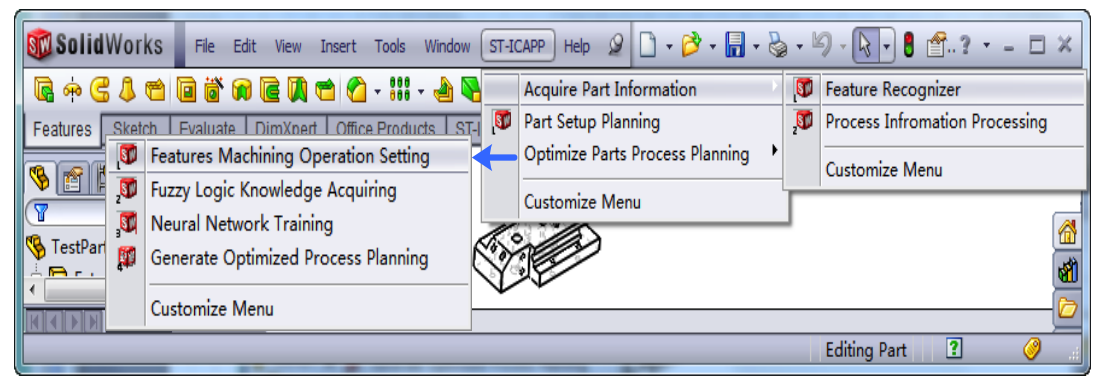

Fig. (8). ST-ICAPP menu in Solidworks 2011.

parts, the left side and the right side according to the cutting point.

(b) Copy the left side of parent 1 to form the left side of child 1. According to the order of operations in parent 2 , the operator constructs the right side of child 1 with operations of parent 2, whose IDs are the same as operations of the right side in parent 1.

(c) The role of these parents is exchanged in order to generate another offspring child.

\section{(3) Mutation}

The mutation operator proposed in this work randomly selects some individuals. The positions of two codes in each individual are swapped randomly. Example of the mutation operation is shown in Fig. (7).

In this work, a new operator is proposed to check the feasibility of the string obtained. If any string violates the constraints, the string is considered infeasible and then the total score is given a very high value so that it will not appear in the next generations. The infeasible solutions are expressed by a penalty:

$$
\text { Fitness }_{\text {(infeasible solution) }}=\alpha
$$

where $\alpha$ is a positive number as large as possible.

Step 5: Do steps 3 and 4 cyclically until the terminating condition is satisfied.

\section{IMPLEMENTATIONS OF ST-ICAPP SYSTEM}

ST-ICAPP system is based on Solidworks 2011 CAD platform by VB.NET programming language. All of user interfaces or dialogs are generated in Solidworks. ST-ICAPP adopts Microsoft Access database system to store feature information, which can be easily accessed later by downstream modules to develop working steps and ISO 14649 part program. In order to keep pace with Solidworks 2011, ST-ICAPP is used as a menu of Solidworks using the Dynamic Link Library (DLL), as shown in Fig. (8). A part model can be edited and the general information and the technological information needed in the process planning can be viewed and edited by clicking the pull-down menus.

This research work applies the object-oriented approach to build the product data model and corresponding design and process planning function models. The model data used in the developed system is based on the application objects defined in STEP AP 224 and ISO 14649(STEP-NC). The objects contain explicit high-level part data in terms of feature attributes, tolerances, material specifications, technological information, part administrative data, machining operations, cutting tools, etc.

In order to illustrate the application of ST-ICAPP, an example part is given as follows.

Fig. (9) shows a part model, design features and machining features by Feature Recognizer. The part's machining features are recognized by Feature Recognizer pull-down menu, the process information can be edited by Process information processing pull-down menu.

Setup planning based on manufacturability of all generated machining features are divided into four groups as shown in Fig. (10) by clicking Part Setup planning menu.

To insure the generated process planning optimization, ST-ICAPP system adopts the intelligent technology as described in the above section. Fig. (11) gives the generated optimal process route. 


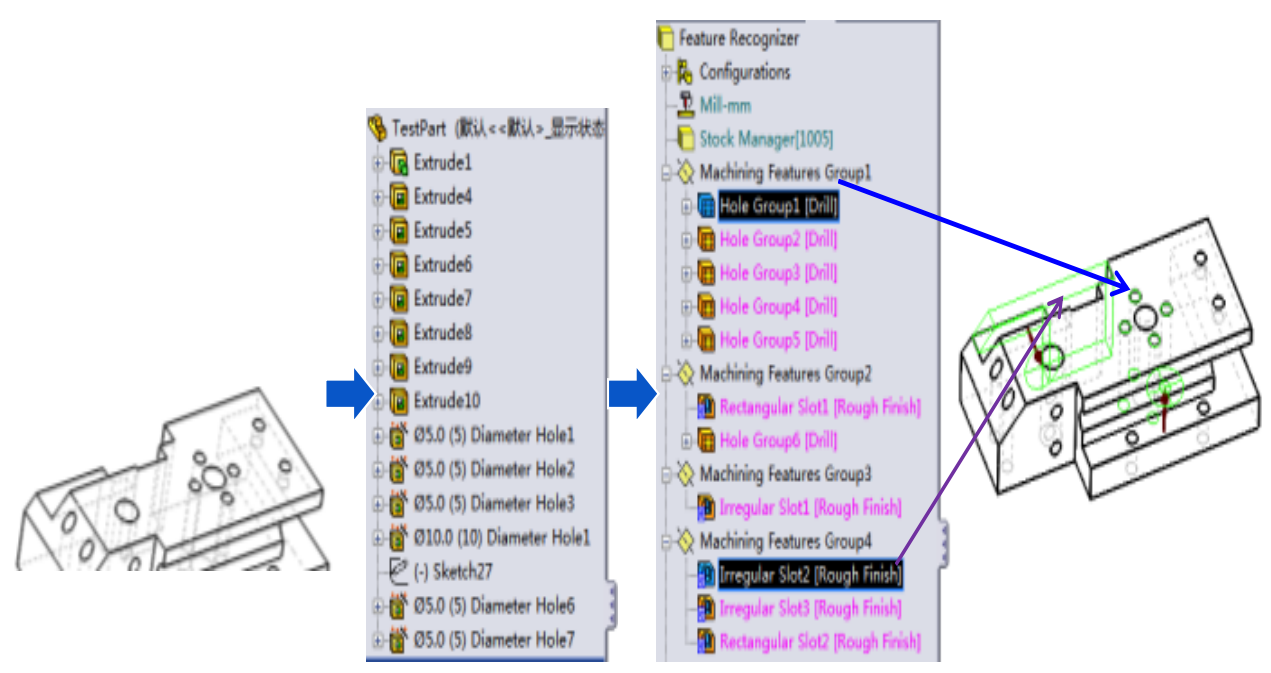

Fig. (9). A part model and recognized machining features.

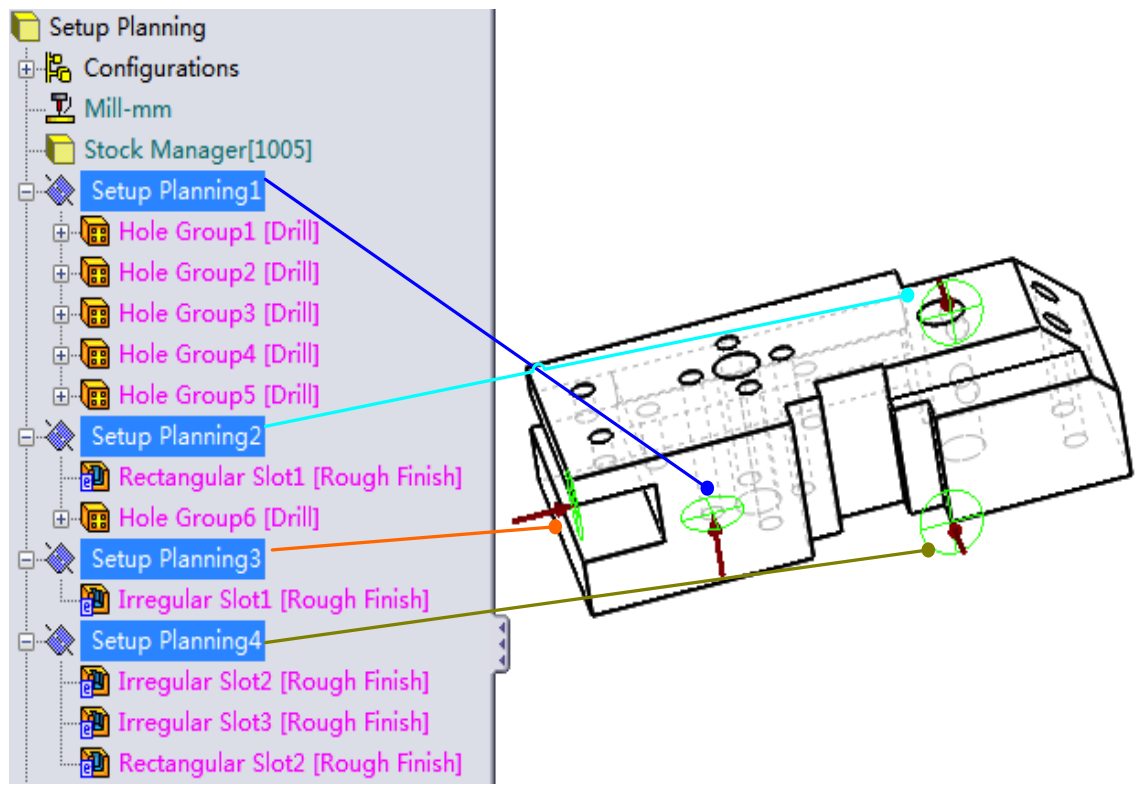

Fig. (10). Setup planning based on part manufacturability.

\section{CONCLUSION}

In the present work, the ISO 14649(STEP-NC) standards and artificial intelligent techniques are applied in the development of ST-ICAPP system. ST-ICAPP system consisted of Acquire Part Information, Part Setup Planning, Optimize Parts Process Planning and STEP-NC Machining Program. By ST-ICAPP, a physical STEP-NC file containing the part manufacturing information, the most suitable setup order and the optimal process route considering a lot of related manufacturing parameters can be obtained. The key technologies of implementing ST-ICAPP system, such as feature recognition, setup planning based manufacturability and the process planning optimization are carried out in detail. A hybrid approach based on artificial intelligent techniques (i.e. neural networks, fuzzy logic and genetic algorithm) is adopted as the inference engine in the proposed ST-ICAPP system.
Artificial neural networks have been used to select machining operations, cutting tools, and machine tools. Several fuzzy logic models have been developed to select machining parameters for machining operations, cutting tool materials, and workpiece material combinations. Optimization of process planning is carried out by a GA algorithm. The hybrid approach has improved the flexibility of the proposed STICAPP system, which can be trained to deal with new knowledge. Using these methodologies described above, the proposed ST-ICAPP system is developed in Solidworks 2011 platform adopted by VB.NET programming language. In the end, the implementation of ST-ICAPP system is given by an example part. The example result proves the possibility of the proposed ST-ICAPP system. The research has presented guidance for the development of the STEP-NC oriented process planning. 


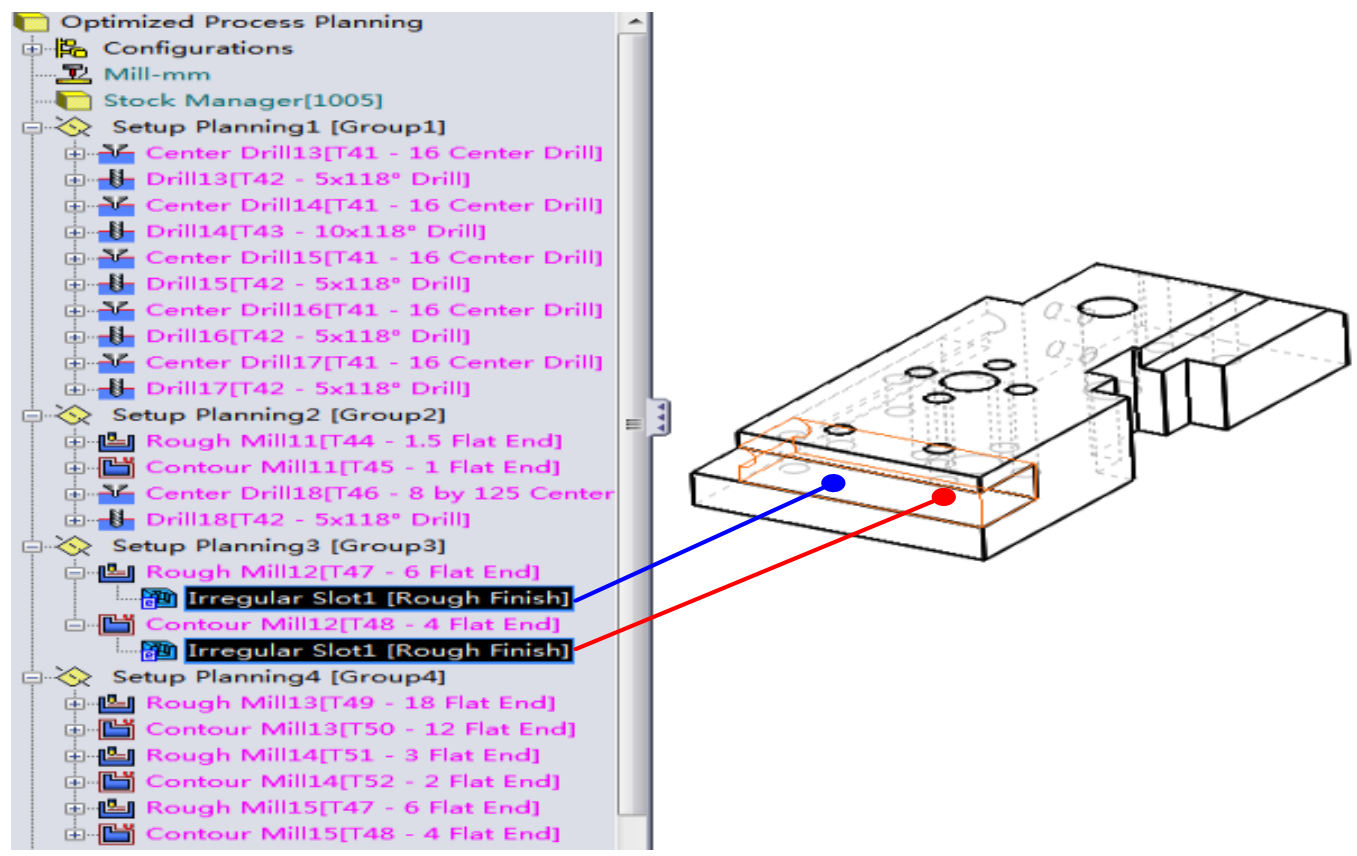

Fig. (11). The generated optimal process route for an example part.

\section{CONFLICT OF INTEREST}

The author confirms that this article content has no conflict of interest.

\section{ACKNOWLEDGEMENTS}

This research is supported by Innovation Program of Shanghai Municipal Education Commission (14YZ158), Shanghai Young Teachers program in University (ZZSDJ12008), Cultivating project of Shanghai Dianji University(12C110),Startup Research Fund from Shanghai Dianji University (13C415).

\section{REFERENCES}

[1] X. W. Xu and Q. He, "Striving for a total integration of CAD, CAPP, CAM and CNC", Int. J. Robotics Comput. Integrat. Manufact., vol. 22, no. 1, pp. 101-109, 2002.

[2] International Standards Organization, ISO 14649-1. Data model for computerized numerical controllers: part 1 -overview and fundamental principles, 2003.

[3] S. H. Suh and S. U. Cheon, "A Framework for an Intelligent CNC and Data Model", Int. J. Adv. Manufact. Technol., vol. 19, no. 10, pp. 727-735, 2002.

[4] S. T. Newman, R. D. Allen, and R. S. U. Rosso Jr, "CAD/CAM solutions for STEP-compliant CNC manufacture," Int. J. Comput. Integrat. Manufact., vol. 16, no. 7-8, pp. 590-597, 2003.

[5] X. Zhang, R. Liu, A. Nassehi and S. T. Newman, "A STEPcompliant process planning system for CNC turning operations", Int. J. Robot. Comput.-Integrat. Manufact., vol. 27, no. 2, pp. 349356, 2011

[6] F. Ridwan, X. Xu, G. Liu, "A framework for machining optimization based on STEP-NC", Int. J. Intell. Manufact., vol. 23, no. 3, pp. 423-441, 2012.

[7] X. Xu, P. Klemm, F. Proctor and S. H. Suh, "STEP-compliant process planning and manufacturing", Int. J. Comput. Integrat. Manufact., vol. 19, no. 1, pp. 491-494, 2006.
[8] S. Kumar, A. Nassehi, S. T. Newman, R. D. Allen and M. K. Tiwari, "Process control in $\mathrm{CNC}$ manufacturing for discrete components:A STEP-NC compliant framework," Int. J. Robot. Comput. Integrat. Manufact., vol. 23, no. 6, pp. 667-676, 2007.

[9] R. L. Liu, C. R. Zhang, S.T.Newman and A. Nassehi, "A STEP-NC programming system for prismatic parts", J. Mat. Sci. Forum, vol. 532-533, pp. 1108-1111, 2007.

[10] X. W. Xu and S. T. Newman, "Making CNC machine tools more open, interoperable and intelligent-a review of the technologies", Int. J. Comput. Indust., vol. 57, no. 2, pp. 141-152, 2006.

[11] A. Nassehi, S. T. Newman and R. D. Allen, "STEP-NC compliant process planning as an enabler for adaptive global manufacturing", Int. J. Robot. Comput.-Integrat. Manufact., vol. 22, no. 5-6, pp. 456-467, 2006.

[12] A. Mokhtar and O. F. Valilai, "Developing a STEP-Compliant multiagent on an interoperable and integrated $\mathrm{CAD} / \mathrm{CAM}$ platform”, Int. J. Manufact. Eng., vol. 2013, pp. 1-9, 2013.

[13] M. G. Marchetta and R. Q. Forradellas, "An artificial intelligence planning approach to manufacturing feature recognition", Int. $J$. Comput.-Aided Design, vol. 42, pp. 248-256, 2010.

[14] Babic, N. Nesic and Z. Miljkovic, "A review of automated feature recognition with rule-based pattern recognition", Int. J. Comput. Indust., vol. 59, no. 4, pp. 321-327, 2008.

[15] X. Xu, L. Wang and S. T. Newman, "Computer-aided process planning: a critical review of recent developments and future trends", Int. J. Robot. Comput.-Integrat. Manufact., vol. 24, no. 1, pp. 1-31, 2011

[16] X. Zhang, A. Nassehi and S. T. Newman, "Feature recognition from CNC part programs for milling operations", Int. J. Adv. Manufact. Technol., vol. 70, no. 1-4, pp. 397-412, 2014.

[17] M. P. Bhandarkar and R. Nagi, "STEP-based feature extraction from STEP geometry for Agile Manufacturing”, Int. J. Comput. Indust., vol. 41, no. 1, pp. 3-24, 2000.

[18] H. C. Zhang and E. H. Lin, "A hybrid-graph approach for automated setup planning in CAPP", Int. J. Robot. Comput.-Integrat. Manufact., vol, 15, no. 1, pp. 89-100, 1999

[19] X. G. Ming and K. L. Mak, "Intelligent setup planning in manufacturing by neural networks based approach", Int. J. Intell. Manufac., vol. 11, pp. 311-331, 2000.

[20] M. Gizaw, A. M. B. A. Rani, and Y. Yusof, "Turn-mill process plan and intelligence machining operations selection on STEP", $A i-$ san J. Scientif. Res., vol. 6, no. 2, pp. 346-352, 2013. 
[21] S. V. Wong, A. M. S. Hamouda, and M. A. E. Baradie, "Generalized fuzzy model for metal cutting data selection", Int. J. Mat. Proces. Technol., vol. 89-90, pp. 310-317, 1999.

[22] M. Salehia and R. T. Moghaddam "Application of genetic algorithm to computer-aided process planning in preliminary and de- tailed planning," Int. J. Eng. Appl. Artif. Intell., vol. 22, no. 8, pp. 1179-1187, 2009.

[23] Z. W. Bo, L. Z. Hua and Z. G. Yu, "Optimization of process route by Genetic Algorithms," Int. J. Robot. Comput.-Integrat. Manufact., vol. 22, no. 2, pp. 180-188, 2006.

Received: September 16, 2014

Revised: December 23, 2014

Accepted: December 31, 2014

(C) Ouyang Hua-bing; Licensee Bentham Open.

This is an open access article licensed under the terms of the Creative Commons Attribution Non-Commercial License (http://creativecommons.org/licenses/ by-nc/4.0/) which permits unrestricted, non-commercial use, distribution and reproduction in any medium, provided the work is properly cited. 